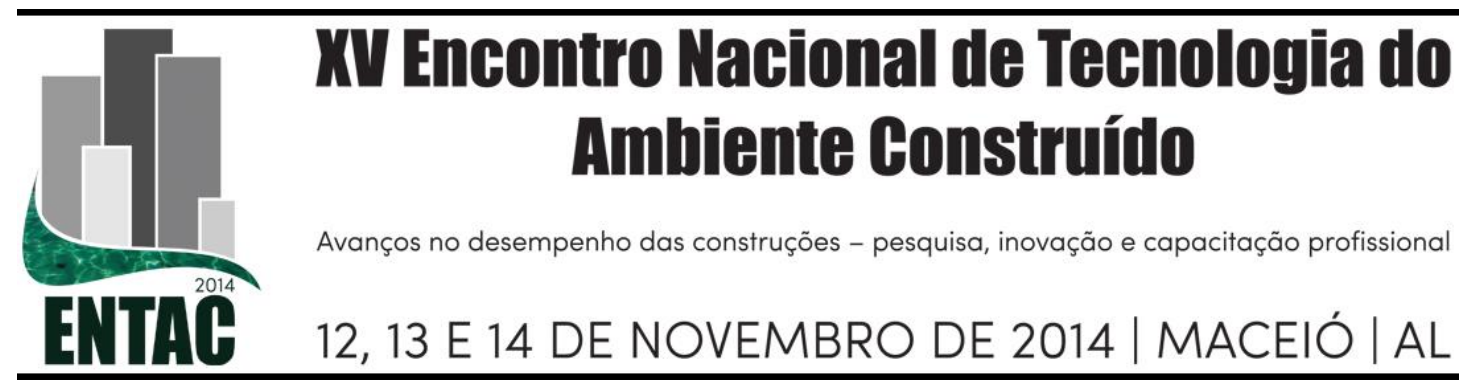

\title{
EFEITOS DE TELHADOS VEGETADOS NO CONFORTO TÉRMICO DE PEDESTRES: SIMULAÇÃO PELO SOFTWARE ENVI-MET
}

ROSSETI, Karyna de Andrade Carvalho (1); NOGUEIRA, Marta Cristina de Jesus Albuquerque (2); CALLEJAS, Ivan Júlio Apolonio (3); DURANTE, Luciane Cleonice (4); KUHNEN, Igor Antonio (5); NOGUEIRA, José de Souza (6)

(1) UFMT, (65) 9972-1125, e-mail: karyna.rosseti@gmail (2) UFMT, e-mail: mcjanp@gmail.com (3) UFMT, e-mail: ivancallejas@ig.com.br (4) UFMT, e-mail:luciane.durante@hotmail.com (5)UFMT, email: igkuhnen@gmail.com (6) UFMT, e-mail: nogueira@ufmt.br

\begin{abstract}
RESUMO
Os benefícios da vegetação no clima urbano podem ser observados não só na área vegetada, mas também no seu entorno. Uma das possibilidades para incorporação da vegetação no contexto urbano é a transformação dos telhados tradicionais dos edifícios em telhados vegetados. O objetivo deste trabalho foi avaliar os efeitos de telhados vegetados no conforto térmico dos pedestres, com simulação pelo software ENVI-Met, na cidade de Cuiabá, MT, localizada em clima tropical continental. A metodologia compreendeu etapas de levantamento microclimático e espacial da área de estudo, modelagem e calibração do modelo, simulação computacional e análise estatística dos resultados. O levantamento microclimático foi realizado por meio de transecto móvel, nos períodos de Janeiro a Março/2013 (quente úmido) e Julho a Setembro/2013 (quente seco). O levantamento espacial foi realizado pela aerofotogrametria, na estação fotogramétrica digital E-foto, complementada por observações in loco. A simulação foi realizada no software ENVI-Met, durante os períodos seco e úmido do ano, em quatro cenários diferentes: cenário real e com incorporação do telhado vegetado em 10, 50 e 100\% dos telhados. Os impactos mais expressivos foram verificados nos cenários com 50 e 100\% de cobertura vegetada, nos quais houve redução superior a 0,5 pontos na escala do PMV, nos quintais. Considerando estes efeitos, os resultados evidenciam que o telhado vegetado se apresenta como estratégia passiva para o planejamento urbano microclimático em climas quentes.
\end{abstract}

Palavras-chave: clima urbano, vegetação, telhados verdes.

\begin{abstract}
The benefits of vegetation on urban climate can be observed not only in the vegetated area, but also in its surroundings. The transformation of traditional buildings roofs in green roofs is one possibility to incorporate vegetation in the urban area. This study evaluated the green roofs effects in the pedestrians' thermal comfort, utilizing ENVI-Met software simulation, at tropical continental climate city (CuiabáMT, Brazil). The methodology included microclimate and spatial survey of the study area, model calibration, computer simulation and statistical analysis. The microclimatic survey was conducted by mobile transect, from January to March/ 2013 (hot humid) and from July to September/2013 (hot dry).The spatial survey was conducted by digital photogrammetric workstation E-photo, complemented by in loco observations. The simulation was performed in ENVI-Met software, during wet and dry periods of the year, in the real scenario and with vegetation incorporation scenarios by 10, 50 and 100\% of the surround roofs area. The most significant results were observed in the 50\% and 100\% scenarios with green roof cover, in which occurred reduction more than 0.5 points in the PMV scale at the backyards. Considering these effects, the results show that the green roof is a passive microclimate strategy for urban planning in hot climate cities
\end{abstract}

Keywords: urban climate, vegetation, green roofs. 


\section{INTRODUÇÃO}

O processo de urbanização modifica a paisagem natural, provocando alterações nas propriedades aerodinâmicas e radiativas da superfície, nas propriedades térmicas do substrato e nas propriedades hidrológicas da superfície e do solo. Além disso, as emissões antropogênicas de calor e poluentes e as alterações nos padrões de evaporação da superfície, modificam as propriedades térmicas e radiométricas da atmosfera urbana (OKE, 1982; HUANG et al., 2009).

O efeito da vegetação no clima urbano já vem sendo investigado há algum tempo. Os benefícios podem ser observados não só na área que contém a vegetação, mas também no seu entorno, demonstrando sua eficácia em reduzir os efeitos da ilha de calor (UHI) (DIMOUDI; NIKOLOPOULOU, 2003).

Em se tratando de áreas urbanas, cerca 20 a 25\% de suas superfícies constituem-se de telhados (AKBARI et al., 2003). Sua utilização como área vegetada pode dar origem a muitos benefícios, tanto na redução dos efeitos da UHI, quanto na melhoria da qualidade do ar, gestão de águas pluviais, aumento da biodiversidade e melhorias no conforto do pedestre (OBERNDORFER et al., 2007).

Este artigo teve por objetivo geral avaliar os efeitos de telhados vegetados na sensação térmica do pedestre, em Cuiabá-MT. O objetivo específico foi analisar cenários com proporções de 10, 50 e 100\% de ocupação dos telhados pela vegetação, às 6,13 e $20 \mathrm{~h}$.

\section{METODOLOGIA}

As seguintes etapas metodológicas foram percorridas no desenvolvimento da pesquisa: definição da zona urbana de estudo; caracterização microclimática da área de estudo utilizando-se de medições para fins de validação do modelo; realização de levantamento espacial da área de estudo utilizando-se de aerofotogrametria; simulação das características morfológicas reais da área de estudo para fins de calibração do software ENVI-Met; criação de arquivo de configuração de entrada do modelo com descrição de cada parâmetro; elaboração de cenários com a incorporação do telhado vegetado em diferentes proporções de ocupação e verificação dos efeitos de cada cenário no conforto térmico dos pedestres.

\subsection{Escolha da zona urbana de estudo}

Com base em Callejas et al. (2011), identificaram-se as regiões residenciais menos adensadas na cidade de Cuiabá, que juntamente com a análise das características topográficas, conduziram à escolha do Jardim Califórnia como área de estudo (Figura 1a). Além disso, a região escolhida não possui muitas variações altimétricas na topografia, considerando que a versão 3.1 do ENVI-met somente simula terrenos planos, não trabalha bem com topografias complexas e não admite a modelagem de áreas muito extensas.

\subsection{Caracterização do microclima na área de estudo}

Os dados microclimáticos para configuração do arquivo de entrada do ENVI-Met foram obtidos na estação de referência (83362), do Aeroporto Marechal Rondon, em Várzea Grande-MT, cidade vizinha à Cuiabá.

Para a validação dos dados gerados pelo ENVI-Met o levantamento microclimático foi realizado na Subcamada Rugosa do Dossel Urbano, definida como a região situada abaixo da altura média dos edifícios. Os dados permitiram verificar a sensibilidade do 
modelo em explicar as particularidades climáticas desta camada urbana. O levantamento foi realizado de forma a demonstrar o comportamento térmico da área de estudo em condições de tempo atmosférico ideal (OKE,1982).

O período de medição foi definido tendo como base as características macroclimáticas da região, na qual ocorrem dois períodos distintos: quente-úmido, de novembro a abril, e quente-seco, de maio a outubro. A medição dos dados microclimáticos referentes à estação quente-úmida ocorreu nos dias 9, 12 e 14 de março e na estação quente-seca, a medição ocorreu nos dias 6, 9 e 12 de agosto, às $06 \mathrm{~h}, 14 \mathrm{~h}$ e $20 \mathrm{~h}$.

Os horários de medição foram definidos com base no comportamento diário da ilha de calor urbana, tendo como parâmetro o nascer do sol, momentos após o pico solar e algumas horas após o pôr do sol, momento no qual se observa a ocorrência da ilha de calor positiva na cidade (CALLEJAS et al. 2011).

Para o levantamento da variável temperatura do ar utilizou-se sensor de temperatura ambiente, modelo S-04K, da Instrutherm. A variável umidade relativa do ar foi medida com termo higrômetro digital portátil, modelo HT-260, da mesma marca. A radiação solar foi obtida com medidor de energia solar, modelo MES-100, da Instrutherm.

O levantamento foi realizado por meio de transecto com veículo automotivo. O termômetro digital portátil foi fixado no vidro do carro e o sensor de temperatura ambiente foi fixado a aproximadamente $1,30 \mathrm{~m}$ do solo, protegido por abrigo ventilado. Os demais equipamentos eram manipulados no momento das medições de dados.

Determinaram-se nove pontos de medição (Figura 1b), nos quais o veículo permanecia parado o tempo de medição (cerca de $1 \mathrm{~min}$ ). O levantamento foi realizado em um tempo total de $20 \mathrm{~min}$.

Figura 1 -Localização do Bairro Jardim Califórnia em Cuiabá-MT (a) e pontos de medição das variáveis microclimáticas (b)

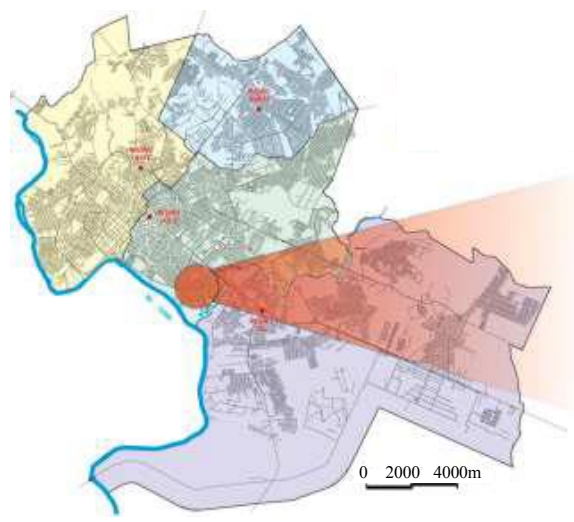

(a)

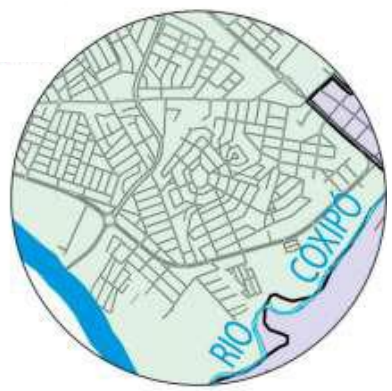

Fonte: Adaptado de Rosseti (2012).

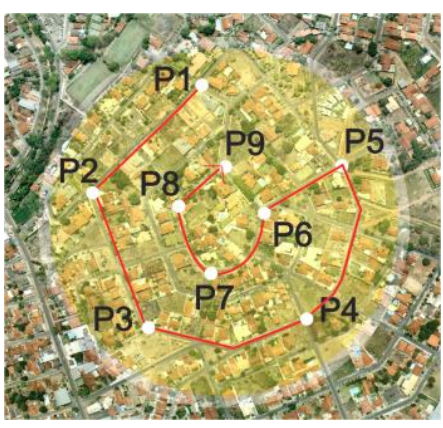

(b) 


\subsection{Caracterização espacial da área de estudo}

O levantamento das variáveis espaciais foi realizada por meio de observações in loco e levantamento aerofotogramétrico na Estação Fotogramétrica Digital E-foto, do Programa de Pós-graduação em Geomática, Universidade do Estado do Rio de Janeiro.

A partir da identificação das cores, opacidade, rugosidade e tipologia dos materiais, o albedo efetivo dos cânions e coberturas, exigidos pelo modelo ENVI-Met, foram obtidos através de pesquisa bibliográfica (SAILOR; FAN, 2002) e experimentação. A identificação das espécies, alturas e exata posição da vegetação presente na área de estudo, basearam-se em observações realizadas in loco. Maior detalhamento pode ser obtido em Rossetti (2013).

\subsection{Modelagem com ENVI-Met}

O modelo numérico microclimático ENVI-met é um modelo tridimensional, não hidrostático, criado por Michael Bruse (BRUSE; FLEER, 1998). A dimensão dos grids foi definida tendo como parâmetro a melhor reprodução das características da área de estudo (calçadas, canteiros, árvores) com o máximo de abrangência territorial, sendo de $2 \mathrm{~m}$. A escala de trabalho foi definida pela necessidade de eliminar possíveis efeitos de borda e permitir a observação dos efeitos do telhado vegetado em maior escala, sem comprometer o tempo de obtenção dos resultados e dificultar a dinâmica computacional do processo de simulação. Optou-se pelo cenário intermediário do ENVI-Met (180x180x30 grids).

Foi necessária a delimitação da área de modelagem dentro da área de estudo. A área modelada totalizou $129.600 \mathrm{~m}^{2}$, dentre os quais $39.600 \mathrm{~m}^{2}$ são referentes aos nesting grids, que configuraram a borda do modelo (Figura 2a). O posicionamento dos receptores baseou-se na localização dos pontos de medição das variáveis microclimáticas, obtidas durante o transecto móvel. Considerando a delimitação da área para adequação à escala do ENVI-Met, somente quatro pontos, dos nove levantados pelo transecto móvel, foram abrangidos pelo modelo (Figura $2 \mathrm{~b}$ ).

\section{Figura 2 - Área de modelagem (a) e localização dos receptores no modelo (b)}

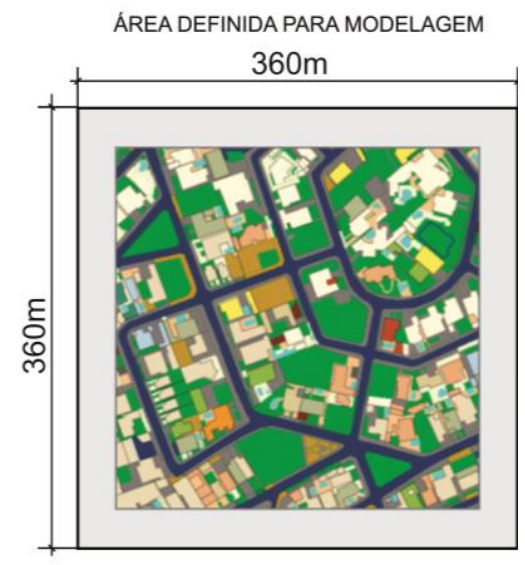

(a)

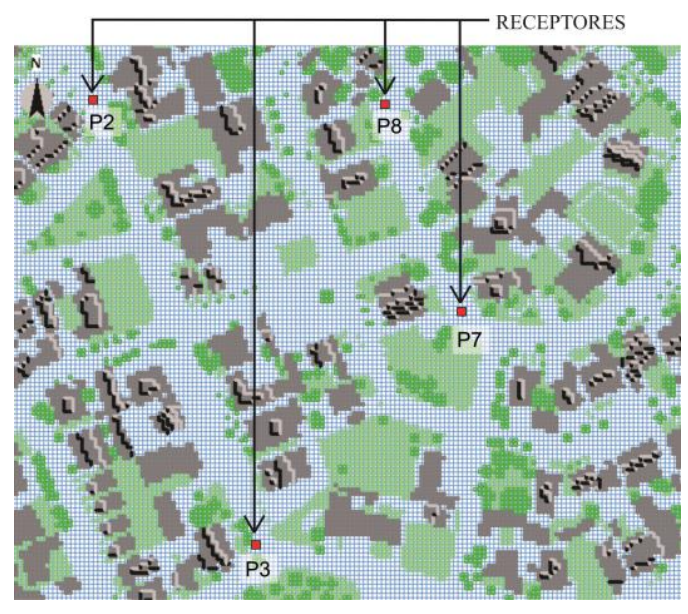

(b) 
O modelo foi caracterizado pela criação de três cenários com proporções de $10,50 \mathrm{e}$ $100 \%$ de ocupação dos telhados pela vegetação, a partir da área total de telhados presente no cenário real, evitando-se os efeitos da concentração da vegetação em uma porção específica da área de estudo. Considerando a exposição solar a que as plantas estarão sujeitas acima dos telhados, optou-se pela utilização de espécies gramíneas do tipo C4 para a composição dos telhados vegetados.

Para comparar estatisticamente os resultados encontrados nos diferentes cenários do estudo utilizou-se a estatística de Kruskal-Wallis, que comprova ou não a existência de diferenças significativas nos valores de PMV obtidos no local de estudo pela incorporação do telhado vegetado.

\subsection{Definição dos parâmetros para análise do conforto dos pedestres}

O ENVI-Met avalia o índice Predictian Mean Vote (PMV) de Fanger, adaptando-o para o ambientes externos estabelecidos por Jendritzky (1993), a partir dos parâmetros de entrada definidos no arquivo de configuração do modelo e das variáveis modeladas pelo software no cenário de estudo, de forma a incorporar o complexo de radiação externa ao cálculo, incluindo na análise os efeitos da radiação direta, difusa e refletida pelos pavimentos e edificações.

Para o cálculo da sensação térmica, foram consideradas: idade 30 anos, altura $1,75 \mathrm{~m}$, peso $70 \mathrm{~kg}$, isolamento térmico das roupas de 0,5 clo e taxa metabólica de $150 \mathrm{~W} / \mathrm{m}^{2}$ correspondente à caminhada em superfície plana a $0,5 \mathrm{~m} / \mathrm{s}$ (ISO, 2005).

Os resultados apresentados pelo modelo ainda devem ser validados para climas tropicais, uma vez que o mesmo utiliza parâmetros de conforto definidos para climas temperados e subtropicais. Assim, os resultados devem ser somente analisados para fins do objetivo a que se destina o presente artigo.

A distribuição espacial dos efeitos da incorporação da vegetação no conforto térmico, medida ao nível do pedestre (1,6m do solo), nos dois períodos do ano, para os três cenários, foi apresentada em mapas por meio de uma escala cromática obtida pelas diferenças entre o PMV do cenário real e dos cenários com telhado vegetado.

\subsection{Análise estatística}

As variáveis utilizadas para a validação do modelo foram temperatura e a umidade do ar. A estatística adotada para avaliar a distância entre os valores das séries temporais medidas e preditas pelo modelo ENVI-Met foi a medida dos erros de predição (SAVI, 2006), sendo elas: o Coeficiente de Correlação $r^{2}$, a Raiz Quadrada do Erro Médio RMSE, o Erro Médio Absoluto MAE e o Desvio das Médias MBE.

\section{RESULTADOS}

\subsection{Validação}

Os indicadores estatísticos do modelo ENVI-Met para as variáveis temperatura e umidade nas estações quente-seca e quente-úmida são apresentados no Quadro 1. Observa-se que o $\mathrm{R}^{2}$ se apresenta elevado para as duas variáveis nos dois períodos, estando somente a umidade relativa do ar, com $\mathrm{R}^{2}$ inferior a 0,91. Quando se trata desta variável, no período úmido, os valores de RMSE, MAE e MBE são elevados, evidenciando que o modelo ENVI-Met subestima seus valores, em todos os horários, 
neste período do ano. O modelo consegue reproduzir, com elevada precisão, a variável de temperatura do ar, sendo mais bem prevista no período seco.

\section{Quadro 1 - Indicadores de desempenho do modelo ENVI-Met}

\begin{tabular}{|c|c|c|c|c|c|c|c|c|}
\hline \multirow{2}{*}{$\begin{array}{c}\text { Variável/ } \\
\text { Período }\end{array}$} & \multicolumn{4}{|c|}{ Temperatura } & \multicolumn{4}{c|}{ Umidade } \\
\cline { 2 - 9 } & $\mathbf{R}^{\mathbf{2}}$ & RMSE & MAE & MBE & $\mathbf{R}^{\mathbf{2}}$ & RMSE & MAE & MBE \\
\hline Seco & 0,9163 & 3,8520 & 3,6144 & $-3,0156$ & 0,8153 & 2,7275 & 4,2106 & $-2,2469$ \\
\hline Úmido & 0,9720 & 4,8500 & 4,8269 & $-4,8269$ & 0,8378 & 14,316 & 14,315 & $-14,315$ \\
\hline
\end{tabular}

A regressão linear evidencia correlação positiva entre as variáveis medidas e estimadas pelo modelo, indicando que elevações na temperatura (Figuras 3a e 3b) e na umidade (Figuras $4 \mathrm{a}$ e $4 \mathrm{~b}$ ) medidas são acompanhadas por elevações na temperatura e na umidade estimadas.

Figura 3 - Dispersão da temperatura do ar estimada versus modelada, período úmido (a) e seco (b)

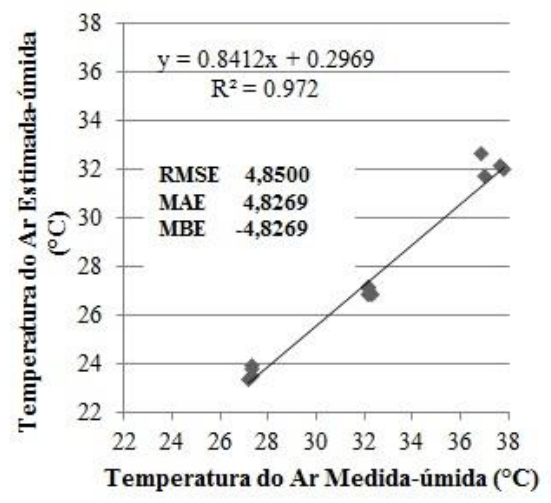

a)

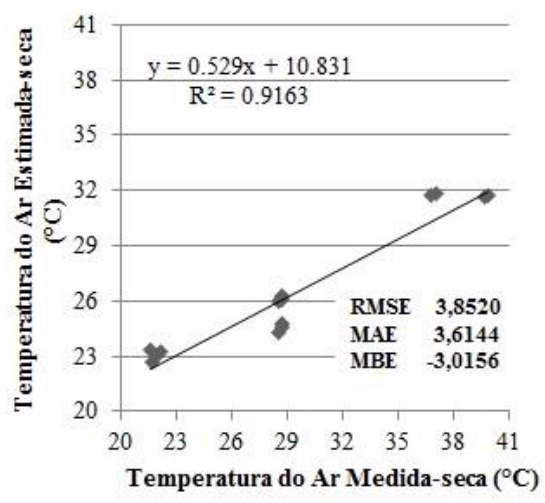

b)

Figura 4 - Dispersão da umidade relativa do ar estimada versus modelada, período úmido (a) e seco (b)

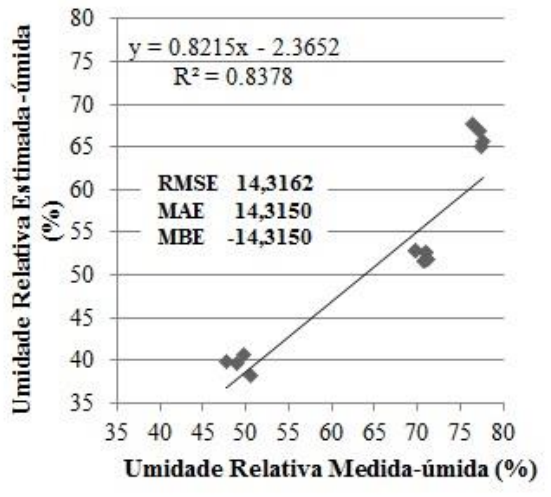

a)

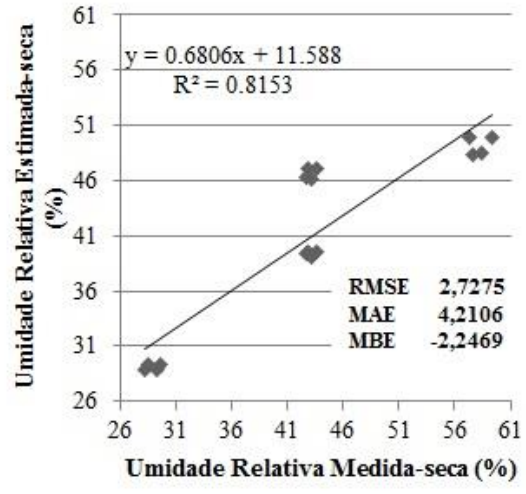

b) 
Os resultados apresentaram correlação forte e positiva entre os resultados medidos e modelados $\left(\mathrm{R}^{2}=0,97\right.$ e 0,84 para temperatura e umidade, respectivamente), podendo-se inferir que o modelo ENVI-Met apresenta-se como uma ferramenta adequada para previsão microclimática do cenário proposto, possibilitando a sua utilização na estimativa dos efeitos do telhado vegetado nesta camada urbana. A dispersão dos pontos em torno da reta de regressão reforça os indicadores de desempenho ilustrados no Quadro 1.

\subsection{Efeitos do Telhado Vegetado}

Com exceção do cenário com incorporação de vegetação em $10 \%$ dos telhados, a estatística de Kruskal-Wallis para a variável PMV indicou que existem diferenças significativas entre os cenários pesquisados, em todos os horários.

Às $06 \mathrm{~h} 00 \mathrm{~min}$, no período seco, foram observadas elevações na escala de 0,05 pontos no PMV em 0,6, 3,4 e 8,8\% dos votos, o que causou modificação da sensação térmica, de "confortável" para "ligeiramente quente", em aproximadamente 0,2, 1,8 e 3,3\% dos votos nos cenários com 10, 50 e 100\% de telhados vegetados, respectivamente. Também se observaram reduções maiores que 0,05 pontos no PMV nos cenários. No entanto, estas não modificaram a sensação térmica. No período úmido, observou-se comportamento semelhante, mas inferior ao período seco, com elevações superiores a 0,05 pontos no PMV, em 0,2, 1,9 e 7,2 dos votos, modificando a sensação térmica de "confortável" para "ligeiramente quente" em aproximadamente 0,1, 0,4 e 1,0 dos votos nos cenários com 10, 50 e 100\% de telhados vegetados, respectivamente. Também se observaram reduções maiores que 0,05 pontos no PMV nos cenários deste período, que não modificaram a sensação térmica. Os reduzidos incrementos nos valores de PMV observados no cenário com $10 \%$ dos telhados com vegetação explica o fato de não haver diferenças significativas em relação ao cenário real, observado no teste estatístico. Estas elevações nos valores de PMV, característicos deste horário, em todos os cenários, demonstram a capacidade do sistema de cobertura vegetada em manter a sua temperatura por um período superior ao sistema de coberturas existente. Este comportamento não pode considerado prejudicial à manutenção do conforto em regiões de clima tropical devido à ocorrência de valores negativos no PMV neste horário, que com a elevação não se distanciaram da situação de conforto.

Às 13h00min, no período seco, foram observadas reduções mais expressivas que nos demais horários, na escala de 0,5 pontos no PMV, em 0,6, 2,8 e 6,4\% dos votos, o que modificou a sensação térmica de "muito quente" para "ligeiramente quente" em aproximadamente $0,1,0,2$ e $0,8 \%$ dos votos nos cenários com 10,50 e $100 \%$ de telhados vegetados, respectivamente. Destaca-se que houve reduções na escala de até 1 ponto no PMV em regiões localizadas nas proximidades dos telhados com vegetação em 0,4, 1,8 e 4,1 dos votos nos cenários com 10, 50 e 100\%. Foram observadas elevações na ordem de 0,05 pontos no PMV nos cenários que, no entanto, não impactaram na modificação da sensação térmica. No período úmido, observou-se comportamento semelhante ao período seco, com reduções na escala de 0,5 pontos no PMV, em 0,6, 2,8 e $6,6 \%$ dos votos, o que causou a modificação da sensação térmica de "muito quente" para "ligeiramente quente" em até $0,15 \%$ dos votos nos cenários com 10, 50 e 100\% de telhados vegetados, respectivamente. Destaca-se que não se observaram reduções na escala de 1 ponto no PMV. Também se observaram elevações na escala de 0,05 pontos no PMV nos cenários deste período que, no entanto, não modificaram a sensação térmica. A maior amplitude das modificações provocadas pelo telhado vegetado neste horário em todos os cenários justifica as diferenças destacadas pelo teste estatístico. 
Às 20h00min, no período seco, diferentemente das $06 \mathrm{~h} 00 \mathrm{~min}$, foram observadas reduções na escala de 0,05 pontos no PMV, em $0,1,0,5$ e 8,8\% dos votos, nos cenários com 10,50 e $100 \%$ de telhados vegetados, respectivamente, que não causaram modificação da sensação térmica. Foram observadas elevações na ordem de 0,05 pontos no PMV nos cenários, que também não modificaram a sensação térmica. No período úmido, observou-se comportamento semelhante ao seco, com reduções na escala de 0,05 pontos no PMV, em 0,1, 0,5 e 6,2\% dos votos, o que, neste caso, causou a modificação da sensação térmica de "muito quente" para "ligeiramente quente" em 0,4, 1,3 e 2,5\% dos votos nos cenários com 10,50 e 100\% de telhados vegetados, respectivamente. Também se observaram elevações na escala de 0,05 pontos no PMV nos cenários deste período, que, no entanto, não modificaram a sensação térmica. Os reduzidos incrementos nos valores de PMV observados no cenário com $10 \%$ dos telhados com vegetação explica o fato de não haver diferenças significativas em relação ao cenário real, observado no teste estatístico.

As Figuras 5 e 6 apresentam os efeitos provocados pela incorporação da vegetação no conforto térmico, nas estações úmida e seca, respectivamente. A escala adotada para as diferenças abrangeu o intervalo onde foram observadas maiores frequências de alterações no PMV (-0,2 a 0,05), abrangendo mais de $90 \%$ dos resultados.

Figura 5 - Efeitos da vegetação no PMV durante os períodos úmido.

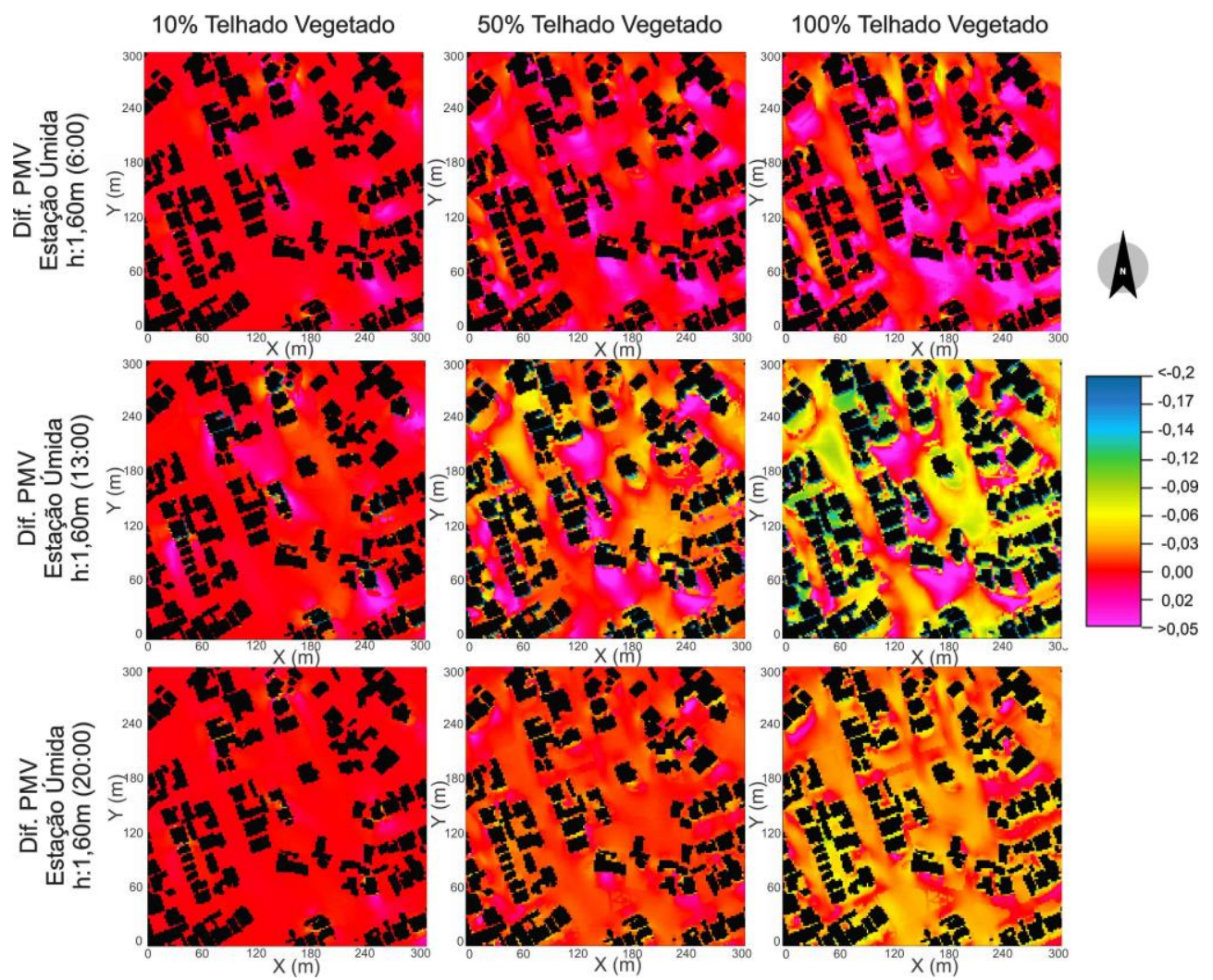

Às 6h00min, nos dois períodos do ano, o telhado vegetado provocou elevações no PMV nos quintais dos edifícios, em todos os cenários. As reduções no PMV apresentam-se nos espaços ocupados pelas vias e calçadas de forma pouco expressiva, sendo mais evidente no cenário com 100\% de telhados vegetados (cores laranjadas e amarelas). 
Às $13 \mathrm{~h} 00 \mathrm{~min}$, foram observadas maiores alterações no PMV, principalmente nos cenários com 50 e 100\% de telhados vegetados. Nestes cenários, nota-se a redução dos valores de PMV acima de 0,12 pontos (cores azuis e verdes). Estas alterações são mais evidentes na região dos quintais em relação aos espaços das ruas e calçadas.

Às 20h00min, nota-se a predominância das cores laranja e amarela nos cenários com 50 e $100 \%$ de telhados vegetados, respectivamente, indicando a redução dos valores de PMV. Estas alterações são mais evidentes na região dos quintais em relação aos espaços das ruas e calçadas. No cenário com $10 \%$ estas alterações não se apresentam evidentes nos mapas, reforçando os resultados da análise estatística.

De uma forma geral o telhado vegetado provocou maiores interferência no período seco. Esta característica se deve principalmente pelo efeito de evapotranspiração provocado pela vegetação dos telhados que foi capaz de alterar as configurações higrotérmicas do ar, principalmente nas suas proximidades. Devido à elevada quantidade de vapor d'água presente no ar no período chuvoso, o fenômeno da evapotranspiração do telhado vegetado é menos perceptível.

\section{Figura 6 - Efeitos da vegetação no PMV durante o período seco.}

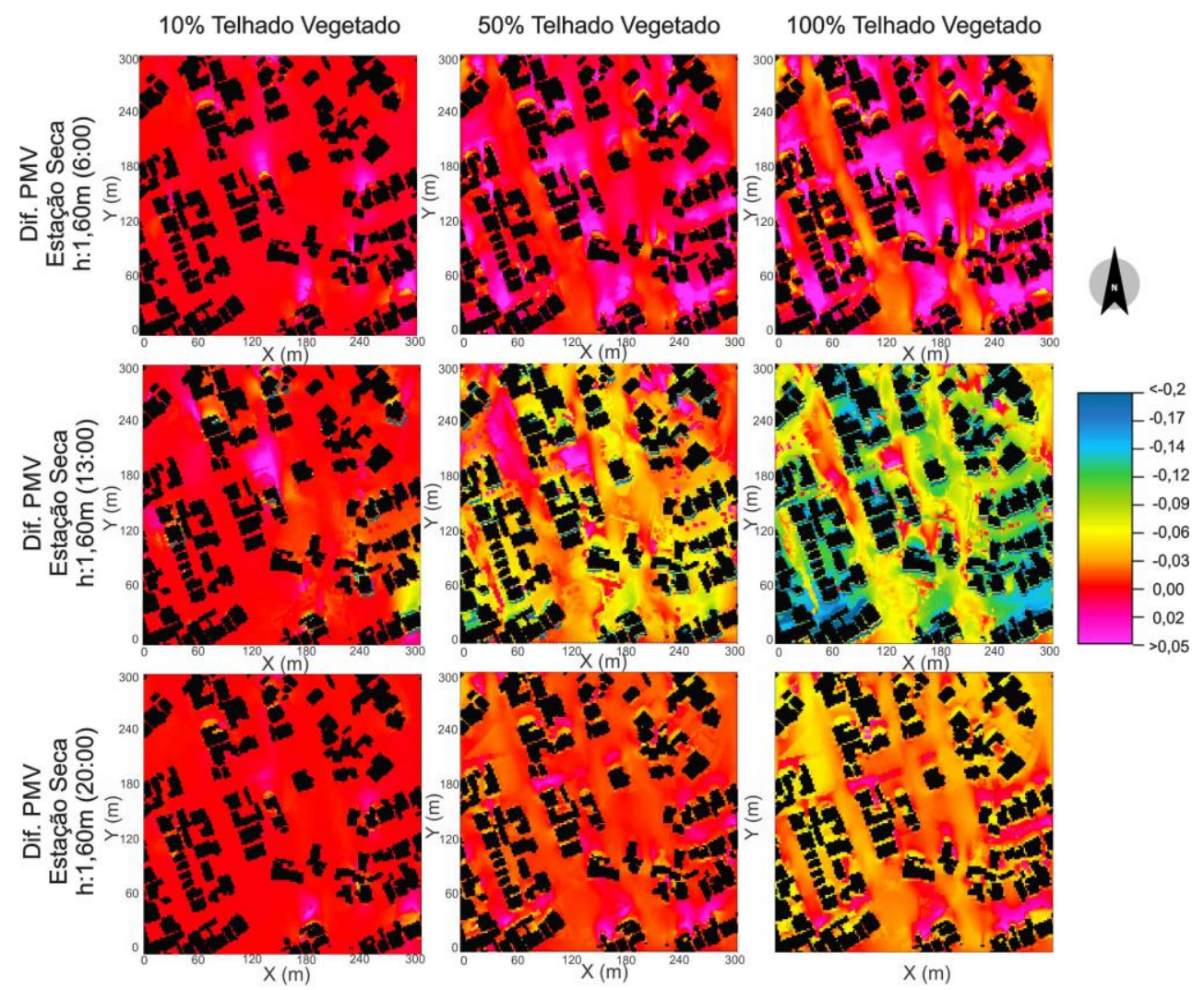

\section{CONCLUSÕES}

Às 06h00min, nos períodos seco e úmido, a incorporação dos telhados vegetados não melhorou a sensação de conforto térmico dos pedestres, elevando o PMV em regiões dos quintais, em todos os cenários.

Às 13 h00min, no período seco e úmido, houve reduções na escala de até 1 ponto no PMV (de "muito quente" para "ligeiramente quente") em regiões localizadas nas proximidades dos telhados com vegetação, nos cenários de 50 e 100\%. 
Às 20h00min, no período seco, a incorporação dos telhados vegetados reduziu o PMV, alterando a sensação térmica de "muito quente" para "ligeiramente quente", com maior evidencia na região dos quintais que no espaço das ruas e calçadas. No cenário com $10 \%$ e no período úmido, o impacto não foi significativo.

\section{REFERENCIAS}

AKBARI, H.; ROSE, S.L.; TAHA, H. Analyzing the land cover of an urban environment using high-resolution orthophotos. Landscape and Urban Planning, v. 63, p. 1-14, 2003.

BRUSE, M.; FLEER, H. Simulating surface-plant-air interactions inside urban environments with a three dimensional numerical model. Environmental Modeling \& Software, v.13, p. 373-384, 1998.

CALLEJAS, I. J. A.; OLIVEIRA, A. S.; SANTOS, F. M. M.; DURANTE, L. C.; NOGUEIRA, M. C. J. A.; ZEILHOFER, P. Relationship between land use/cover and surface temperatures in the urban agglomeration of Cuiabá-Várzea Grande, Central Brazil. Journal of Applied Remote Sensing, v. 5, p. 0535691-05356915, 2011.

CALLEJAS, I. J. A.; DURANTE, L. C.; Oliveira, A. S.; SANTOS, F. M. M.; NOQUEIRA, M. C. J. A. Estudo comparativo de temperatura e umidade entre meio urbano e rural. In: XI Encontro Nacional de Conforto no Ambiente Construído e VII Encontro Latino Americano de Conforto no Ambiente Construído, 2011, Buzios-RJ. Anais... Encontro Nacional e Encontro Latino-Americano de Conforto no Ambiente Construído, 2011. v. 1.

CLEUGH, N. A.; OKE, T. R. Suburban-rural energy balance comparisons in summer for Vancouver, BC. Boundary-Layer Meteorol, v. 36, p. 351-369, 1986.

DIMOUDI, A.; NIKOLOPOULOU, M. Vegetation in the urban environment: Microclimatic analysis and benefits. Energy and Buildings, v. 35, p. 69 -76, 2003.

HUANG, S.; TANIGUCHI, M.; YAMANO, M.; WANG, C. Detecting urbanization effects on surface and subsurface thermal environment - a case study of Osaka. Science of the Total Environment, v. 407, p. 3142-3152, 2009.

INTERNATIONAL ORGANIZATION FOR STANDARDIZATION. ISO 7730: Ergonomics of the thermal environment: analytical determination and interpretation of thermal comfort using calculation of the PMV and PPD indices and local thermal comfort criteria. Switzerland, 2005.

JENDRITZKY, G. Human biometeorology, Part I. Experientia, v. 49, n. 9, p. 733-740, 1993.

OBERNDORFER, E.; LUNDHOLM, J.; BASS, B.; COFFMAN, R. R.; DOSHI, H.; DUNNETT, N.; GAFFIN, S. R.; KÖHLER, M.; LIU, K. K. Y.; ROWE, B. Green roofs as urban ecosystems: ecological structures, functions and services. BioScience, v. 57, n. 10, p. 823-833, 2007.

OKE, T. R. The energetic basis of the urban heat island. Quarterly Journal of the Royal Meteorological Society, v. 108, p. 1-24, 1982.

ROSSETI, K. A. C. Efeitos do uso de telhados vegetados em ilhas de calor urbanas com simulação pelo software ENVI-met. 2013. 253 f. Tese (Doutorado em Física Ambiental) Instituto de Física da Universidade Federal de Mato Grosso, Cuiabá, 2013.

SAILOR, D. J.; FAN, H. Modeling the diurnal variability of effective albedo for cities. Atmospheric Environment, v. 36, n. 4, p. 713-725, 2002.

SAVI, M. A. Dinâmica não-linear e caos. Editora E-papers, 2006. 